\title{
THE FIDELITY EVOLUTION FOR QUANTUM CHAOTIC KICKED KERR-LIKE NONLINEAR OSCILLATOR AND DISTANCE EFFECT OF THE INITIAL WAVE FUNCTIONS
}

\author{
J. K. Kalaga ${ }^{1}$, A. Kowalewska-Kudłaszyk ${ }^{1}$, W. Leoński ${ }^{2}$, V. Cao Long ${ }^{2}$ \\ ${ }^{1}$ Nonlinear Optics Division, Department of Physics, Adam Mickiewicz University, \\ Umultowska, 85, 61-614 Poznań, Poland \\ ${ }^{2}$ Quantum Optics and Engineering Division, Institute of Physics, University of Zielona Góra, \\ ul. Prof. A. Szafrana 4a, 65-516 Zielona Góra, Poland
}

(Received November 06, 2010; received in final form January 11, 2011)

\begin{abstract}
We discuss a system of the Kerr-like quantum nonlinear oscillator excited by a series of ultrashort external pulses. These coherent excitations, along with the nonlinearity involved in the system, can lead to the quantum chaotic behaviour. For that model, we calculate the fidelities of the wave function which describe the system's dynamics. We show that such defined fidelity and its decay rate can be applied as an indicator of the quantum chaotic behaviour appearance. We also discuss the problem of how the initial distance between the two close wave functions can influence the fidelity changes.
\end{abstract}

Key words: quantum chaos, fidelity, entropy, Kerr nonlinear oscillator.

PACS number(s): 05.45.Mt, 42.50.Dv

\section{INTRODUCTION}

There are many problems concerning classical dynamics of the physical systems that are treated as chaotic ones. For these chaotically behaving models one can talk about a chaotic motion when the system (whose dynamics is described by the nonlinear equations) is sensitive to initial conditions. In consequence, the system's trajectories defined in the phase space become unstable and errors in the initial data grow exponentially. As a result, the final state of the system is unpredictable. The methods for analysing such a situation are well developed. For instance, one can numerically determine the chaotic behaviour of the system analysing the Lyapunov exponents. However, for quantum systems, as a result of the linearity of the Schrödinger equation (for this case, the system's dynamics is governed by the unitary evolution operator), small changes in the initial state do not cause indefinite changes in the final state of the considered system. Therefore, standard methods used for problems of classical chaos are not useful for such cases.

In this paper, we shall concentrate on the method that leads to a description of the quantum dynamics of systems behaving chaotically in the classical limit basing on the time varying fidelity between quantum states. As it has been shown the fidelity can be used as a signature of chaos [1-3]. In fact, the fidelity is a measure of stability of time evolution of quantum states. It is worth noting that the fidelity can be used as a standard parameter which allows a measure of decoherence in the systems applied in quantum information theory. It has been proved that whenever a quantum system begins to behave chaotically, the fidelity decreases exponentially [4-6]. The rate of the fidelity decay has been the subject of several studies and depending on the perturbation character and its strength, various types of that decay have been identified.
It has been shown that for the perturbative regime (which corresponds to small perturbations) the fidelity decay is of the Gaussian type. It is also characteristic of integrable and quasi-integrable classical dynamics. The increase in the value of the perturbation strength leads to a golden rule regime of fidelity decay. Such a decay is exponential [5] with the slope depending on the pertubation strength. In general, one can say that the decay of the quantum fidelity corresponding to the quantum chaotic regions is slower than those for integrable ones.

The main aim of our considerations presented in this paper is an analysis of the fidelity of the time-evolution for the long-time limit, contrary to papers [2,3] where generally the short-time limit was considered. As we shall show, the behaviour of this strictly quantum parameter can indicate whether the considered system is located within the area of regular or chaotic dynamics. Moreover, we shall show that the analysis of the influence of the value of initial divergence between wave-functions can give us the information concerning the character of the system evolution.

For our considerations, we apply the quantum analogue of a kicked Kerr-like nonlinear oscillator (some features of which have been discussed in [7]). As was shown in our previous papers [8,9], this model is a good example of the quantum chaotic systems which can be used effectively as a tool for various considerations of the quantum chaos indicator.

\section{THE MODEL}

We consider the known model comprising the nonlinear Kerr-like oscillator externally driven by a series of ultra-short coherent pulses [7]. This model is often referred to as the "kicked nonlinear oscillator". Moreover, it is known that the classical counterpart of this model 


\section{J. K. KALAGA, A. KOWALEWSKA-KUDŁASZYK, W. LEOŃSKI, V. CAO LONG}

may exhibit regular and chaotic behaviours as well. The character of a such system's dynamics depends on the values of the parameters that describe the system.

The dynamics of the system is governed by the following Hamiltonian (we use the units of $\hbar=1$ ):

$$
\hat{H}=\hat{H}_{N L}+\hat{H}_{K}
$$

where $\hat{H}_{N L}$ describes the evolution of the system between the two subsequent pulses and is defined as

$$
\hat{H}_{N L}=\frac{\chi}{2}\left(\hat{a}^{\dagger}\right)^{2} \hat{a}^{2}
$$

The parameter $\chi$ is a nonlinearity constant, whereas $\hat{a}$ $\left(\hat{a}^{\dagger}\right)$ is a bosonic annihilation (creation) operator.

The Hamiltonian $\hat{H}_{K}$ corresponds to the interaction of the system with a single infinitesimally short external pulse and is given by

$$
\hat{H}_{K}=\varepsilon\left(\hat{a}^{\dagger}+\hat{a}\right) \sum_{k=1}^{\infty} \delta(t-k T)
$$

where $T$ is a time between the two subsequent pulses and $\varepsilon$ is the strength of the external field - nonlinear oscillator interaction. As was mentioned above, the pulseduration is assumed to be extremely short. In fact, the time-duration of the pulse is much smaller than the time $T$. Therefore, we can model such series of pulses by a sum of the Dirac-delta functions.

Since, we neglect all damping processes, the system's evolution can be examined by unitary evolution operators. They are defined on the basis of the above Hamiltonians. Thanks to the fact that the pulses are extremely short, the whole evolution can be divided onto two stages. The first one corresponds to the "free" nonlinear evolution, whereas the second is related to the action of the pulses. Thus, for the period of time between the two subsequent external pulses the evolution operator can be expressed as:

$$
\hat{U}_{N L}=e^{-i \chi T \hat{n}(\hat{n}-1) / 2},
$$

whereas that for the interaction with a single pulse is related to

$$
\hat{U}_{K}=e^{-i \varepsilon\left(\hat{a}^{\dagger}+\hat{a}\right)} .
$$

We assume that the system is prepared initially in the vacuum state $|\psi(0)\rangle=|0\rangle$. For the time just after the $k$ th external pulse the wave-function describing the state of the system can be determined by action ( $k$ times) of the above operators on $|\psi(0)\rangle$. It can be expressed as:

$$
|\psi(k)\rangle=\left(\hat{U}_{K} \hat{U}_{N L}\right)^{k}|\psi(0)\rangle .
$$

It should be stressed that in fact we perform a quantum mapping procedure.

As we shall compare the dynamics of the quantum system with its classical counterpart we need to determine the classically regular and chaotic motion regions. To do it we perform the procedure presented in $[7,9]$ to find the classical regular and chaotic regions. Within this procedure we define the parameter $\alpha$ that is a classical counterpart of the bosonic annihilation operator. The recurrence mapping formula for this parameter has the following form $[7,9]$ :

$$
\alpha_{k+1}=\left(\alpha_{k}-i \varepsilon\right) e^{-i\left(\chi\left|\alpha_{k}-i \varepsilon\right|^{2}\right) T} .
$$

In fact, the quantity $\left|\alpha_{k}\right|^{2}$ denotes the classical system's energy and corresponds to the mean number of photons $\left\langle\hat{a}^{\dagger} \hat{a}\right\rangle$ for the quantum case. Assuming that for the case discussed here $T=\pi$ and $\chi=1$ and offering the discussion analogous to that presented in [7], we have determined the regions of the classically regular and chaotic motion regions. Thus, we have stated that for $0<\varepsilon<0.344$ and $0.356<\varepsilon<0.47$ the system exhibits regular behaviour whereas for $\varepsilon>0.47$ and $\varepsilon \approx 0.35$ a chaotic evolution can be observed. If the value of $\varepsilon$ exceeds $\sim 0.47$ considerably, we can say that the system reaches "deep chaos" regime. The determination of these conditions will help us to further discuss the results obtained for the quantum system.

\section{THE RATE OF FIDELITY DECAY}

Similarly to $[1,2]$ we define the fidelity $F(k)$ as an indicator of the quantum chaotic system's behaviour. However, one should keep in mind that papers $[2,3]$ were devoted to the short-time behaviour of the fidelity whereas in the present paper we shall concentrate on its longtime evolution. We believe that such an attempt is more appropriate, it is more related with the philosophy of deterministic chaos investigations.

Thus, we define the fidelity between the wave-function corresponding to the unperturbed system and that for the system affected by some tiny perturbation. To introduce such a perturbation we define new unitary evolution operator corresponding to the action of a single external pulse. This operator can be expressed as:

$$
\hat{U}_{K p}=e^{-i(\varepsilon+\Delta)\left(\hat{a}^{\dagger}+\hat{a}\right)}
$$

where we have introduced some tiny perturbation $\Delta \ll \varepsilon$. This operator can be used in the quantum mapping procedure analogous to that defined by eqs.(4-6).

Since the fidelity is defined as

$$
\mathcal{F}(k)=\left|\left\langle\psi_{u}(k) \mid \psi_{p}(k)\right\rangle\right|
$$

we can write the following formula determining its quantum mapping

$$
\mathcal{F}(k)=\left|\left\langle\psi(t=0)\left|\left(\hat{U}_{N L}^{\dagger} \hat{U}_{K p}^{\dagger}\right)^{k}\left(\hat{U}_{K} \hat{U}_{N L}\right)^{k}\right| \psi(t=0)\right\rangle\right| .
$$

Such a defined fidelity parameter allows us to determine the system's characteristics concerning its chaotic or regular behaviours. 


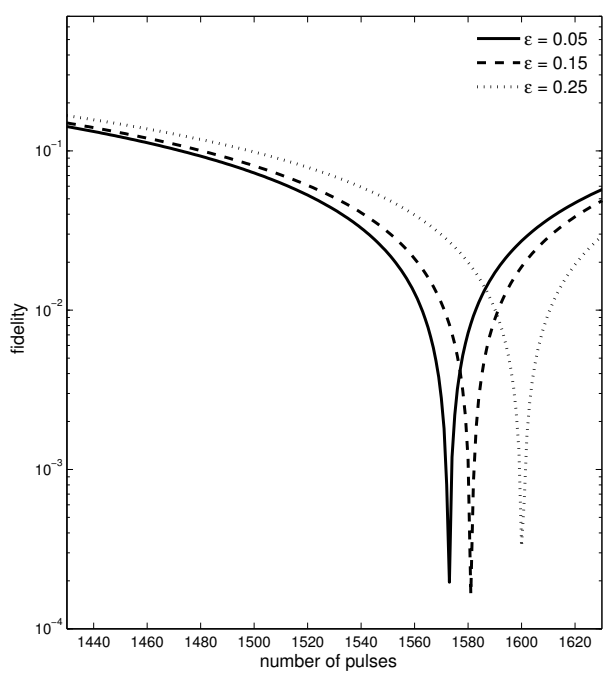

a)

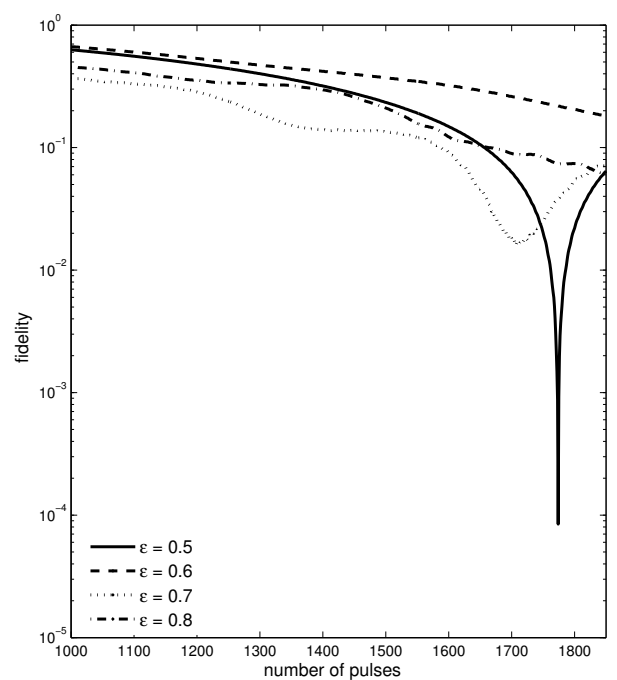

b)

Fig. 1. The fidelity $\mathcal{F}$ versus the number of pulses for various values of the strength of the external pulse for $\Delta=0.001$ : a) regular region for classical system; b) chaotic region for classical system.

It is known that for the short-time regime the fidelity decay is quadratic [2]. However, after some period of time, the character of decay changes considerably and comes to depend on the strength of perturbation. In particular, when the value of the perturbation parameter is much smaller than that describing the strength of the external pulse $(\Delta \ll \varepsilon)$, the decay is of the Gaussian type. However, if we increase the perturbation strength $(\Delta)$, the decay becomes exponential [2].

In this paper, we shall study the case when the decay of the fidelity has the Gaussian character showing how the fidelity decay changes with the varying external pulse's strength $\varepsilon$. Therefore, we intend to concentrate our discussion on fidelity and its decay rates for various values of $\varepsilon$. Thus, from Fig. 1 we can see that the decay rate changes depending on the strength of the external pulse. For regular regions (Fig. 1a) we can observe that the decay rate is smaller if the value of $\varepsilon$ is larger. Moreover, the first minimal value of fidelity (seen in Fig. 1a) is reached earlier if we decrease external coupling. For the chaotic regions (Fig.1b) the situation becomes more complicated. It is difficult to determine for which value of $\varepsilon$ the rate reaches the smallest value, especially if we are inside the deep chaos regime. However, we can see that the system needs more time to reach the first minimum of the fidelity. Moreover, for higher values of the excitation strength instead of the well pronounced minimum we can observe a few shallow ones.

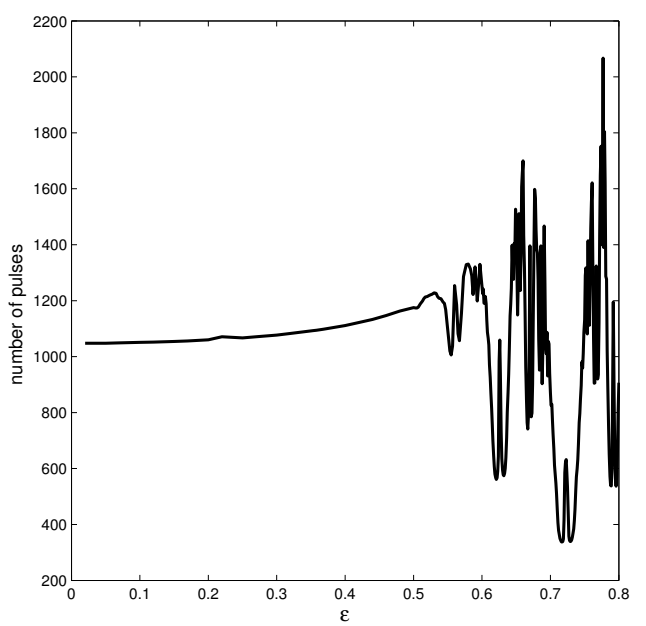

Fig. 2. The number of pules for which fidelity $\mathcal{F}=0.5$ versus the strength of the external pulse $(\Delta=0.001)$.

To show the unpredictable character of the fidelity evolution for the chaotic cases we have plotted (Fig.2) the number of external pulses necessary to achieve by $\mathcal{F}$ the value equal to $1 / 2$ as a function of $\varepsilon$. This number gives us the information about the fidelity decay rate. We see that for the cases when $\varepsilon \lesssim 0.52$ the number of pulses grows slightly. For higher values of $\varepsilon$ it changes its value in a completely chaotic way. Moreover, it should be stressed that if the value of $\varepsilon$ corresponds to the first, narrow chaotic band (for the classical system), we can see the same smooth $\varepsilon$-dependence as for the regular region. So, this chaotic behaviour band is characteristic only for the classical system [6] and it does not appear for the quantum system. However, when the value of $\varepsilon$ corresponds to the "deep chaos" region for the classical system, changes of the rate have an irregular character.

Such behaviour can be observed for various values of the initial perturbation parameter $\Delta$ (Fig.3). However, if we increase the value of $\Delta$, the first maximum is shifted slightly towards higher values of $\varepsilon$. Moreover, the higher values of $\Delta$ we assume (stronger initial perturbation), the lower the curves corresponding to them are located. We see that the number of pulses necessary to get $1 / 2$ by the fidelity is much smaller when the initial divergence $\Delta$ is greater. In addition, for higher values of $\Delta$ and "deep chaos" regime although we observe the rapid changes in the values of the plotted parameter, the character of these changes is not so rapid as for smaller values of $\Delta$. 


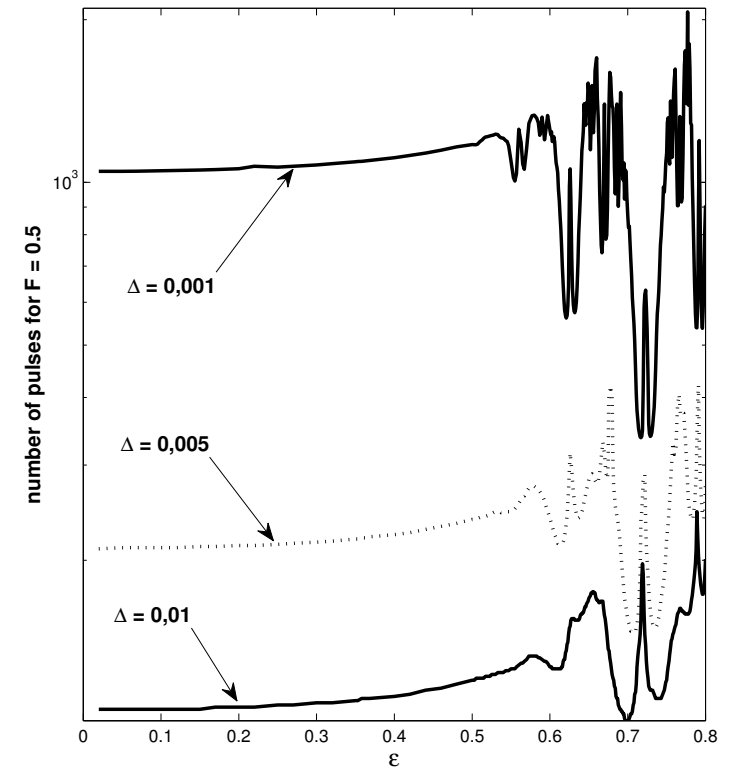

Fig. 3. The number of pules for which fidelity $\mathcal{F}=0.5$ versus the strength of the external pulse for various values of the perturbation parameter $\Delta$.

We see that the character of changes of the rate of fidelity decay depends on the region in which the system evolution is located. The changes of the rate are sensible if the system evolution is characteristic of the "deep chaos" regime. However, if we examine the plots shown in Fig. 2 and 3, we see that the rate is not sensible for narrow chaotic bands and appearances of regular windows.

\section{SUMMARY}

Applying the model of the externally kicked nonlinear Kerr-like oscillator we have shown that the fidelity between wave-functions can be applied as an indicator of quantum chaos. In particular, we have concentrated on the fidelity decay rate. As was shown this rate can be used as an indicator of the chaotic behaviour of the quantum system for the "deep chaos" regime. However, the indicators discussed here are not sensible for sufficiently narrow chaotic bands and for regular windows inside regions of chaos, as well. Moreover, we have shown that the parameters discussed here can exhibit irregular, chaotic behaviour in the classical sense despite their strict quantum nature. The results presented here strengthen our belief that the fidelity (and other parameters defined on its basis) can be a useful tool for further investigations in the field of quantum chaos.

\section{ACKNOWLEDGMENT}

J. K. K. and W.L. acknowledge support from the National Science Centre under Grant No. N N202 195240.
[1] A. Peres, Phys. Rev. A, 30, 1610 (1984).

[2] Y. S. Weinstein, S. Lloyd, C. Tsallis, Phys. Rev. Lett. 89, 214101 (2002).

[3] Y. S. Weinstein, J. V. Emerson, S. Lloyd, D. G. Cory, Quantum Information Processing 1, 439 (2003).

[4] R. A. Jalabert, H. M. Pastawski, Phys. Rev. Lett. 86, 2490 (2001).

[5] P. Jacquod, P. G. Silvestrov, C. W. J. Beenakker, Phys.
Rev. E 64, 055203 (2001).

[6] T. Prosen, Phys. Rev. E 65, 036208 (2002).

[7] W. Leoński, Physica A 233, 365 (1996).

[8] A. Kowalewska-Kudłaszyk, J. K. Kalaga, W. Leoński, Phys. Lett. A 373, 1334 (2009).

[9] A. Kowalewska-Kudłaszyk, J. K. Kalaga, W. Leoński, Phys. Rev. E 78, 066219 (2009).

\title{
ЕВОЛЮЦІЯ “ТОЧНОСТІ" КВАНТОВОГО ХАОТИЧНОГО ЗБУДЖЕНОГО КЕРРІВСЬКОГО НЕЛІНІЙНОГО ОСЦИЛЯТОРА І ВПЛИВ ВІДСТАНІ МІЖ ПОЧАТКОВИМИ ХВИЛЬОВИМИ ФУНКЦІЯМИ
}

\author{
Й. К. Каляга ${ }^{1}$, А. Ковалєвска-Кудлашик ${ }^{1}$, В. Лєоньскі ${ }^{2}$, В. Као Лонг ${ }^{2}$ \\ ${ }^{1}$ Відділ нелінійної оптики, фізичний факультет, Університет Адама Мічкевича, Познань, Польща \\ ${ }^{2}$ Відділ квантової оптики та інженерї, Інститут фізики, \\ Університет Зельоної Гури, Зелъона Гура, Полъща
}

\footnotetext{
У статті розглянуто систему керрівських квантових нелінійних осциляторів, збуджених серією ультракоротких зовнішніх імпульсів. Ці когерентні збудження разом із нелінійністю в системі можуть спричиняти квантову хаотичну поведінку. Для такої моделі розраховано точність відтворення хвильової функції, яка описує динаміку системи. Показано, що так визначена "точність" і швидкість їі розпаду може слугувати індикатором появи квантової хаотичної поведінки. Також обговорено проблему того, як початкова відстань між двома близькими хвильовими функціями може впливати на зміну точності.
} 